\title{
Paper A New Code Family for QS-CDMA Visible Light Communication Systems
}

\author{
Mouad Addad and Ali Djebbari
}

Faculty of Electrical Engineering, Djillali Liabes University of Sidi Bel Abbes, Sidi Bel Abbès, Algeria

https://doi.org/10.26636/jtit.2018.124318

\begin{abstract}
Visible light communication (VLC) is a promising technology for wireless communication networks. Optical code division multiple access (OCDMA) is a strong candidate for VLC-based applications. The predominant source of bit error in OCDMA is the multiple access interference (MAI). To eliminate MAI in synchronous OCDMA, zero cross correlation (ZCC) codes have been proposed. However, synchronization problems and multipath propagation introduce relative non-zero time delays. Therefore, the zero correlation zone (ZCZ) concept was introduced. In this paper, we propose a new method for generating ZCC codes. The proposed construction can accommodate any number of users with flexible Hamming weight. The numerical results obtained show that the proposed codes significantly reduce MAI, compared to $\mathrm{ZCC}$, as well as ZCZ codes.
\end{abstract}

Keywords-CDMA, visible light communication, zero correlation zone, zero cross correlation.

\section{Introduction}

Wireless access technologies have been continuously evolving in response to the rapid increase in the use of mobile devices [1]. The scarcity of radio frequency spectrum is a limiting factor in meeting this demand [2]. In applications where high bandwidth is required, visible light communication (VLC) is a promising technology, complementary to radio frequency systems. A key advantage of VLC is its potential to simultaneously provide energy sufficient lighting and high-speed communication using light emitting diodes (LEDs) [1]-[5]. VLC is being adopted in, to name a few, vehicle-to-vehicle communication [3], indoor positioning [4], and underwater communications [5].

A crucial aspect of a VLC system is the multiple access technique. The optical medium is suited for spread spectrum multiple access (SSMA) communications due to its large bandwidth. Code division multiple access (CDMA) is one class of SSMA, in which many users access a common channel simultaneously through the use of encoding. In an optical CDMA system (OCDMA), the received signal is the superposition of light waves from the individual users.

The performance of OCDMA systems depends on the code set employed. The design of proper codes for OCDMA must take into consideration many criteria, such as: large set size, equal Hamming weight, minimum length, and most importantly low cross correlation properties. Zero cross correlation $(\mathrm{ZCC})$ codes were proposed to eliminate the MAI effect in synchronous OCDMA systems [6], [7]. However, synchronization problems and multipath propagation introduce a relative non-zero time delay. Therefore, zero correlation zone (ZCZ) codes were introduced for the optical quasi-synchronous (QS) CDMA [8].

The remaining content of the paper is organized as follows. New family of ZCC codes is introduced in Section 2. In Section 3, the system model for optical QS-CDMA is given. Performance of the system using various optical codes is evaluated in Section 4. We give our concluding remarks in Section 5 .

\section{Preliminaries}

ZCC code is an orthogonal set of sequences where no overlapping of ones occurs. The code parameters must be flexible, so that it can suit any application: number of users can take any integer and is independent of the code Hamming weight (number of ones in any single code). Let $\mathbf{S}$ be a set with $M$ codes each with length $N$ where $\mathbf{S}=\left\{\mathbf{s}^{1}, \ldots, \mathbf{s}^{i}, \ldots, \mathbf{s}^{M}\right\}$, and $\mathbf{s}^{i}=\left\{s_{1}^{i}, \ldots, s_{l}^{i}, \ldots, s_{N}^{i}\right\}:$

$$
\mathbf{S}=\left[\begin{array}{c}
\mathbf{s}^{1} \\
\vdots \\
\mathbf{s}^{i} \\
\vdots \\
\mathbf{s}^{M}
\end{array}\right]=\left[\begin{array}{ccccc}
s_{1}^{1} & \ldots & s_{l}^{1} & \ldots & s_{N}^{1} \\
\vdots & \ddots & \vdots & \ddots & \vdots \\
s_{1}^{i} & \ldots & s_{l}^{i} & \ldots & s_{N}^{i} \\
\vdots & \ddots & \vdots & \ddots & \vdots \\
s_{1}^{M} & \ldots & s_{l}^{M} & \ldots & s_{N}^{M}
\end{array}\right]
$$

and $s_{l}^{i} \in\{0,1\}$.

The periodic cross-correlation function (CCF) is defined as follows:

$$
R_{\mathbf{s}^{i}, \mathbf{s}^{j}}(\tau)=\sum_{l=1}^{N} s_{l}^{i} s_{(l+\tau) \bmod N}^{j},
$$

when $i=j$ the CCF becomes the auto-correlation function (ACF).

The code set $\mathbf{S}$ is called optical ZCZ set if the correlation functions satisfy [6], [7]:

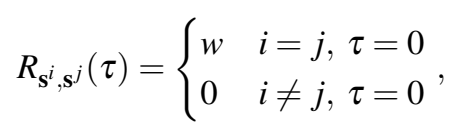


where $w$ represents the code's Hamming weight. The code length for $\mathrm{ZCC}$ codes is $N=M w$.

A code set $\mathbf{S}$ is called $\mathrm{ZCZ}$ if the correlation functions satisfy [8]:

$$
R_{\mathbf{s}^{i}, \mathbf{s}^{j}}(\tau)= \begin{cases}w & i=j, \tau=0 \\ 0 & i \neq j, \tau=0 \\ 0 & 0<|\tau| \leq Z\end{cases}
$$

where $Z$ is the zero-zone length. The code length for $\mathrm{ZCZ}$ codes is $N=M w(Z+1)$.

\subsection{New ZCC Code Family}

In this section, a simple and flexible construction method of ZCC codes is presented. The number of codes as well as the code Hamming weight can be easily adjusted. The construction procedure is as follows. For $w=2$, a starter $\mathrm{ZCC}$ code is obtained as:

$$
\mathbf{Z C C}_{w=2}^{M=2}=\left[\begin{array}{llll}
1 & 1 & 0 & 0 \\
0 & 0 & 1 & 1
\end{array}\right]
$$

To increase the number of codes to $M+1$, a mapping technique is used as:

$$
\mathbf{Z C C}_{w}^{M+1}=\left[\begin{array}{ll}
\mathbf{A} & \mathbf{B} \\
\mathbf{C} & \mathbf{D}
\end{array}\right]
$$

where $\mathbf{A}$ is the original ZCC set of $[M, N], \mathbf{B}$ consists of $[M, w]$ zeros, $\mathbf{C}$ consists of $[1, N]$ zeros, and $\mathbf{D}$ of $\mathrm{a}[1, w]$ ones. Example: by using the mapping technique (6) on ZCC given by (5), we obtain:

$$
\mathbf{Z C C}_{w=2}^{M=3}=\left[\begin{array}{llllll}
1 & 1 & 0 & 0 & 0 & 0 \\
0 & 0 & 1 & 1 & 0 & 0 \\
0 & 0 & 0 & 0 & 1 & 1
\end{array}\right] .
$$

The ZCC code length is $N=w M$.

\section{System Model}

The optical QS-CDMA for VLC system is designed in [8] to support $M$ active users, each containing an information source and a destination. Each user employs a white LED for signal transmission and a photodiode for signal reception. The optical source is assumed to be ideally flat over a bandwidth $\left[\begin{array}{cc}v_{0}-\frac{\Delta_{v}}{2} & v_{0}+\frac{\Delta_{v}}{2}\end{array}\right]$ where $v_{0}$ denotes the optical central frequency and $\Delta_{v}$ the bandwidth. Users are considered to have an equal transmitted and received power. The LED broadband spectrum is divided into $N$ wavelengths, where each user is assigned a set of wavelengths. Each bit " 1 " of the user's assigned code picks a wavelength. When the information is carried on the intensity of light, the signals that modulate the LEDs must be real and nonnegative. Therefore, unipolar codes made up of " 1 " and " 0 " are used.

The main sources of LED-based OCDMA degradation are phase-induced intensity noise (PIIN), shot noise, and thermal noise. PIIN generated at the photodiode output can be suppressed using ZCC and ZCZ codes [6]-[8]. Therefore, only the shot noise and thermal noise are present in the photodiode current. The current variance at the receiver can be expressed as [6], [8]:

$$
\operatorname{Var}(i)=2 e I B+\frac{4 k_{\mathrm{B}} T_{n} B}{R_{L}},
$$

where $e$ is the electron charge, $I$ is the average photocurrent, $B$ is the receiver electrical bandwidth, $k_{\mathrm{B}}$ is the Boltzmann's constant, $T_{n}$ the receiver noise temperature, and $R_{L}$ is the receiver load resistor. The average photo current is given as [8]:

$$
I=\frac{R P_{s r}}{N}\left(w d_{j}+\sum_{i=1, i \neq j}^{M} d_{i} \sum_{l=1}^{N} s_{l-\tau_{i}}^{i} s_{l}^{j}\right)
$$

where $P_{s r}$ is the received power, $d_{j}$ is the desired user's transmitted data bit, $d_{i}$ is the interferer's transmitted bit, and $R$ is the responsivity of the photodiode given as [8]:

$$
R=\frac{\eta e}{h v_{0}}
$$

where $\eta$ is the photo detector's quantum efficiency and $h$ is Planck's constant.

When ZCC and ZCZ codes are used, the desired user's current for a time delay $\tau_{i}=0$ is:

$$
I_{d}=\frac{R P_{s r} w d_{j}}{N}
$$

For quasi-synchronous transmission $\left(\tau_{i} \neq 0\right)$ and worst case scenario $\left(d_{i}=d_{j}=1\right)$, the averaged average current $\bar{I}$ is given as [8]:

$$
\bar{I}=\frac{R P_{s r}}{N}\left(w+\frac{1}{Z} \sum_{\tau_{i}=1}^{Z} \sum_{i=1, i \neq j}^{M} \sum_{l=1}^{N} s_{l-\tau_{i}}^{i} s_{l}^{j}\right) .
$$

The average signal to noise ratio (SNR) for optical QSCDMA system is [8]:

$$
S N R=\frac{I_{d}^{2}}{\operatorname{Var}(i)}=\frac{\left(\frac{R P_{s r} w}{N}\right)^{2}}{\frac{2 e B R P_{s r}}{N}\left(w+\frac{M A I\left(\tau_{i}\right)}{Z}\right)+\frac{4 k_{\mathrm{B}} T_{n} B}{R_{L}}},
$$

where $Z$ is the maximum time delay between all users and $\operatorname{MAI}\left(\tau_{i}\right)$ is the multiple access interference (MAI) defined as:

$$
\operatorname{MAI}\left(\tau_{i}\right)=\sum_{\tau_{i}=1}^{Z} \sum_{i=1, i \neq j}^{M} \sum_{l=1}^{N} s_{l-\tau_{i}^{i}}^{i} s_{l}^{j} .
$$

\section{Performance Analysis}

The MAI is a significant cause of bit decision errors for an optical QS-CDMA system. The correlation properties of codes are crucial to the system's capacity to eliminate MAI and provide reliable communication. In this section, we first investigate MAI reduction of the proposed ZCC 
codes based on time delay, code length, and the number of codes. Next, ZCC codes are compared to ZCZ codes.

In order to evaluate the impact of correlation properties on the code's performance, we compare the proposed ZCC family to two ZCC codes proposed in [6], [7]. The same number of codes $M=10$, code length $N=80$, and Hamming weight $w=8$ were used. From Fig. 1, it is clear that when the time delay between active users increases, MAI also increases in the system. This is because a longer time delay results in higher cumulative correlation function values. With their good correlation properties, the ZCC codes proposed provide lower MAI.

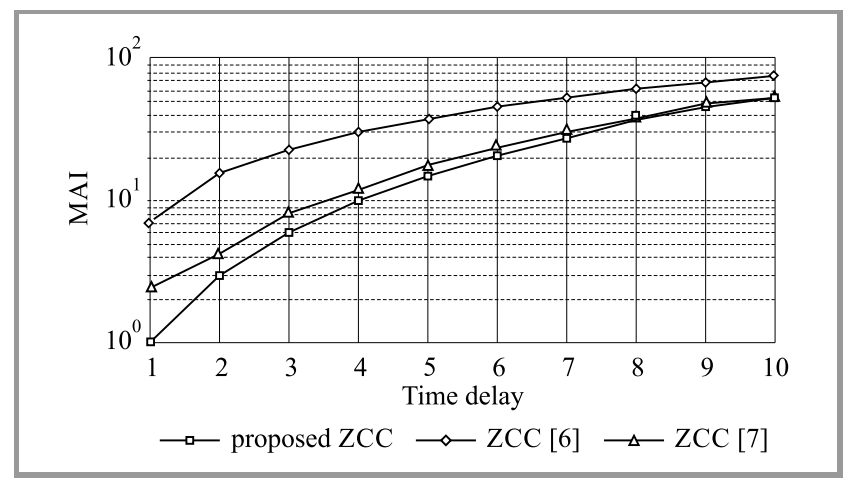

Fig. 1. MAI vs. time delay.

The impact of code length on MAI is analyzed and plotted in Fig. 2. ZCC codes with $M=10$ active users having maximum time delay of $Z=5$ were evaluated. The MAI level increases with code length $N$. Since $M$ is fixed, longer codes can be obtained by increasing the Hamming weight $w$. This results in more ones " 1 " in the code and, consequently, higher correlation values. Note that the proposed ZCC codes not only have the lowest MAI level but also remain constant for code length $N$ higher than 50 .

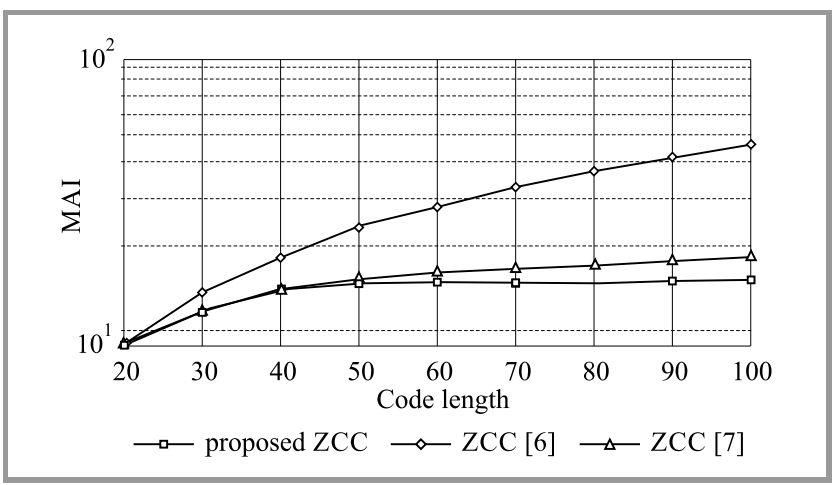

Fig. 2. MAI vs. code length.

Next, the interferer effect on MAI is analyzed. Figure 3 shows MAI values versus the number of active users $M$. ZCC codes with Hamming weight $w=8$, code length $N=$ 10 , and time delay $Z=3$ were evaluated. One can see that admitting more users to the system results in a high level of MAI. Note that the proposed ZCC codes still maintain the lowest values of MAI.

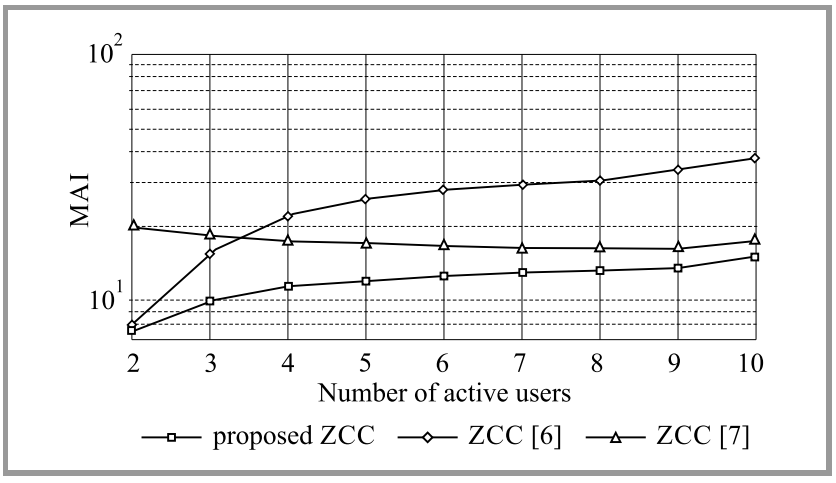

Fig. 3. MAI vs. number of active users.

\subsection{Comparison between ZCC and ZCZ Codes}

Due to their zero-correlation property, ZCZ codes have been extensively studied for radio frequency systems (more details can be found in [9], [10]). In [8], the authors introduced optical ZCZ codes to eliminate MAI in an optical QS-CDMA VLC system. Optical ZCZ codes can eliminate interference $\operatorname{MAI}\left(\tau_{i}\right)=0$ for $\tau_{i} \leq Z$. The SNR for $\mathrm{ZCZ}$ codes is:

$$
S N R=\frac{\left(\frac{R P_{s r} w}{N}\right)^{2}}{\frac{2 e B R P_{s r} w}{N}+\frac{4 k_{\mathrm{B}} T_{n} B}{R_{L}}} .
$$

Since MAI cannot be used to compare ZCC and ZCZ codes performance, BER will be used instead. BER can be computed as follows [8]:

$$
B E R=0.5 \cdot \operatorname{erfc} \sqrt{\frac{S N R}{8}} .
$$

Table 1

\begin{tabular}{|c|c|c|}
\hline Symbol & Quantity & Value \\
\hline$v_{0}$ & $\begin{array}{l}\text { Blue light } \\
\text { center } \\
\text { frequency }\end{array}$ & $480 \mathrm{~nm}$ \\
\hline$\Delta_{v}$ & $\begin{array}{l}\text { Modulation } \\
\text { bandwidth }\end{array}$ & $650 \mathrm{MHz}$ \\
\hline$\eta$ & $\begin{array}{c}\text { Photo detector } \\
\text { quantum } \\
\text { efficiency }\end{array}$ & 0.6 \\
\hline$T_{n}$ & $\begin{array}{c}\text { Receiver noise } \\
\text { temperature }\end{array}$ & $300 \mathrm{~K}$ \\
\hline$R_{L}$ & $\begin{array}{l}\text { Receiver load } \\
\text { resistor }\end{array}$ & $1030 \Omega$ \\
\hline$B$ & $\begin{array}{r}\text { Receiver } \\
\text { electrical } \\
\text { bandwidth }\end{array}$ & $311 \mathrm{MHz}$ \\
\hline$e$ & Electron charge & $1.602189 \cdot 10^{-19} \mathrm{C}$ \\
\hline$k_{\mathrm{B}}$ & $\begin{array}{c}\text { Boltzmann's } \\
\text { constant }\end{array}$ & $1.3806505 \cdot 10^{-23} \frac{\mathrm{J}}{\mathrm{K}}$ \\
\hline$h$ & $\begin{array}{l}\text { Planck's } \\
\text { constant }\end{array}$ & $6.626196 \cdot 10^{-34} \mathrm{~J} \cdot \mathrm{s}$ \\
\hline
\end{tabular}

System parameters 
The parameters used in the computation of analytical results are listed in Table 1 [8].

Figure 4 shows BER performance with respect to the received power $P_{s r}$. ZCC and ZCZ codes with $M=4$ active users and a different time delay were evaluated. The Ham-

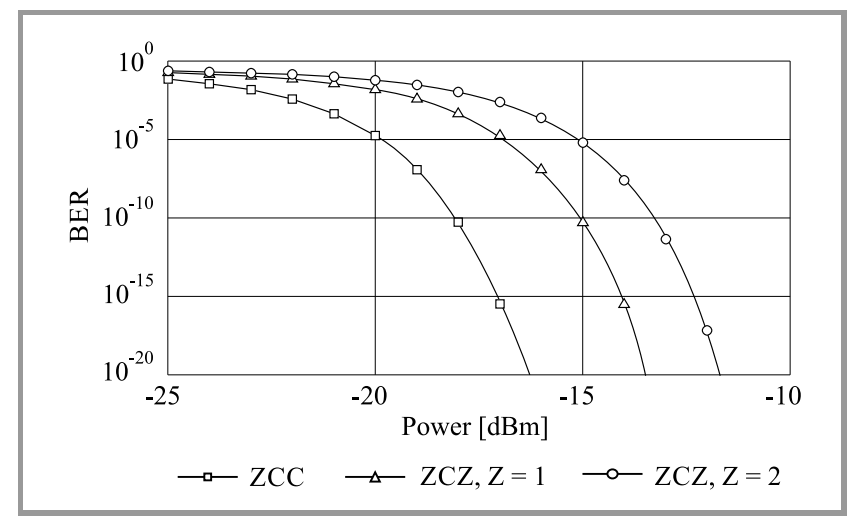

Fig. 4. BER comparison between ZCC and ZCZ codes.

ming weight is $w=3$. We can readily see that ZCC codes outperform ZCZ codes by a large extent. Although ZCZ codes eliminate MAI, their code length is proportional to the zero zone $Z$. A large time delay requires long $Z C Z$ codes which results in poor BER performance.

\section{Conclusion}

In this paper, we evaluate the performance of an optical QSCDMA VLC system. MAI caused by the quasi-synchronous transmission was investigated with respect to ZCC correlation properties, code length, and number of codes in the set. New ZCC codes with a flexible construction and good correlation properties were presented in this paper. It was shown that the proposed ZCC codes have the best performance according to the criteria previously mentioned. It was also shown that ZCZ could eliminate MAI, but their length is not practical and results in poor BER performance. Therefore, we conclude that the new ZCC codes provide robust communication in a QS-CDMA VLC system.

\section{References}

[1] K. Werfli et al., "Experimental demonstration of high-speed $4 \times 4$ imaging multi-CAP MIMO visible light communications", J. of Lightwave Technol., vol. 36, no. 10, pp. 1944-1951, 2018 (doi: 10.1109/JLT.2018.2796503).

[2] A. K. Gupta and A. Chockalingam, "Performance of MIMO modulation schemes with imaging receivers in visible light communication", J. of Lightwave Technol., vol. 36, no. 10, pp. 1912-1927, 2018 (doi: 10.1109/JLT.2018.2795698).

[3] V. T. B. Tram and M. Yoo, "Vehicle-to-vehicle distance estimation using a low-resolution camera based on visible light communications", IEEE Access, vol. 6, pp. 4521-4527, 2018 (doi: 10.1109/ACCESS.2018.2793306).

[4] A. Naz, H. M. Asif, T. Umer, and B. S. Kim, "PDOA based indoor positioning using visible light communication", in IEEE Access, vol. 6, pp. 7557-7564, 2018 (doi: 10.1109/ACCESS.2018.2796623).
[5] F. Miramirkhani and M. Uysal, "Visible light communication channel modeling for underwater environments with blocking and shadowing", IEEE Access, vol. 6, pp. 1082-1090, 2018 (doi: 10.1109/ACCESS.2017.2777883).

[6] T. H. Abd, S. A. Aljunid, H. A. Fadhil, R. A. Ahmed, and N. Saad, "Developpment of a new code family based on SAC-OCDMA system with large cardinality for OCDMA network", Optic. Fiber Technol., vol. 17, no. 4, pp. 273-280, 2011 (doi: 10.1016/j.yofte.2011.04.002).

[7] A. Garadi A. and A. Djebbari, "New technique for construction of a zero cross correlation code", Optik, vol. 123, no. 15, pp. 1382-1384, 2012 (doi: 10.1016/j.ijleo.2011.08.017).

[8] L. Feng, J. Wang, R. Q. Qingyang, and L. Liu, "New design of optical zero correlation zone codes in quasi-synchronous VLC CDMA systems", EURASIP J. on Wireless Communic. and Network., vol. 2015, no. 1, pp. 1-7, 2015 (doi: 10.1186/s13638-015-0360-z).

[9] M. Addad and A. Djebbari, "A ternary zero correlation zone sequence sets construction procedure", Turkish J. of Electr. Engin. and Comp. Sciences, vol. 25, no. 3, pp. 2455-2463, 2017 (doi: 10.3906/elk-1603-115).

[10] M. Addad and A. Djebbari, "Suitable Sequences for Asynchronous MC-CDMA Systems", J. of Telecommun. and Inform. Technol., no. 3, pp. 9-13, 2018 (doi: 10.26636/jtit.2018.118217).

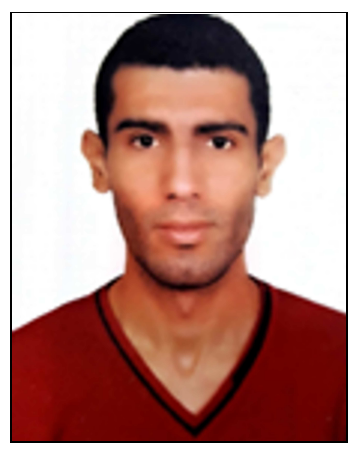

Mouad Addad received his M.Sc. degree in Telecommunications in 2013 from the University of Djillali Liabes In Sidi Bel Abbes, Algeria. He is currently pursuing his Ph.D. degree at the same university. His main research interests include sequences design for communication applications, signal processing, and wireless communication systems over multipath and fading channel.

E-mail: ad-mouad@hotmail.fr

Faculty of Electrical Engineering

Djillali Liabes University of Sidi Bel Abbes

Sidi Bel Abbès, Algeria

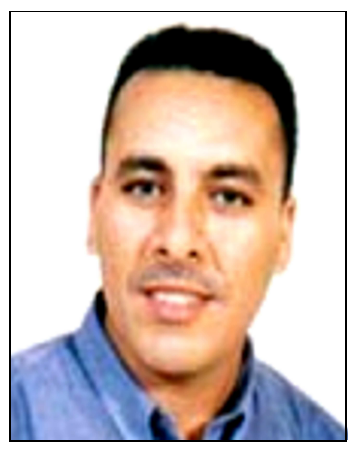

Ali Djebbari received his B.Sc. degree in 1988, from the University of Sciences and Technology of Oran (USTO, Algeria), M.Sc. degree in 1991, from the University of Sidi Bel Abbes, and the Ph.D. degree in 1997, from USTO. He works at the Department of Telecommunications at the University of Sidi Bel Abbes and does his research at the Telecommunications and Digital Signal Processing Laboratory. His research interests include signal processing for telecommunications, communication over multipath and fading channels, wireless networks, channel coding and optical communication networks.

E-mail: adjebari2002@yahoo.fr

Faculty of Electrical Engineering

Djillali Liabes University of Sidi Bel Abbes

Sidi Bel Abbès, Algeria 